\title{
THE FUNDAMENTAL POWER COUPLER PROTOTYPE FOR THE SPALLATION NEUTRON SOURCE (SNS) SUPERCONDUCTING CAVITIES*
}

\author{
I. E. Campisi ${ }^{\dagger}$, E. F. Daly, J. E. Henry, P. Kneisel, W. J. Schneider, M. Stirbet, K. M. Wilson \\ TJNAF, Newport News, VA 23606, USA
}

\section{Abstract}

The SNS linac contains superconducting cavities with $\beta$ .61 and .81 for the acceleration of $\mathrm{H}^{-}$ions. Each of the 6cell cavities, resonant at $805 \mathrm{MHz}$ at $2.1 \mathrm{~K}$, is powered by a $550-\mathrm{kW}$ pulsed klystron via a coaxial coupler. The specifications for the fundamental power coupler (FPC) require that it withstand the full klystron power in full reflection for the duration of the RF pulse $(1.3+0.15$ [decay] ms) at a repetition rate of $60 \mathrm{pps}$ with an average power of $48 \mathrm{~kW}$. The FPC must provide a $\mathrm{Q}_{\mathrm{ext}}$ of $7.3 \mathrm{x}$ $10^{5}$ and $7.0 \times 10^{5}$ for the medium- $\beta$ and high- $\beta$ cavities, respectively. A design derived from proven experience at other laboratories (primarily at KEK, for Tristan and KEK-B cavities at $508 \mathrm{MHz}$ ) has been adopted for the prototypes of the FPCs. The 50- $\Omega$ coaxial line is scaled directly from $508 \mathrm{MHz}$ to the SNS frequency of 805 $\mathrm{MHz}$.

\section{INTRODUCTION}

The Spallation Neutron Source makes use of superconducting RF cavities resonating at $805 \mathrm{MHz}$ in the fundamental $\mathrm{TM}_{010}-\pi$ mode to accelerate $\mathrm{H}^{-}$ions in the main linac from $185 \mathrm{MeV}$ to the full final energy (840$1300 \mathrm{MeV}$ ) [1].

The superconducting cavities, under development at Jefferson Lab [2], must produce accelerating gradients consistent with peak surface electric fields of $27.5 \mathrm{MV} / \mathrm{m}$, or better. In order to power the cavities, coaxial couplers were chosen. The couplers must be able to withstand at least the peak power delivered by the SNS klystrons (shown in Table 1), $550 \mathrm{~kW}$ for a 1.3 -msec pulse length at a repetition rate of 60 pulses per seconds (pps).

Table 1: Coupler requirements

\begin{tabular}{|c|c|c|}
\hline Parameter & Operation & Processing \\
\hline Qext & $7 \times 10^{5}$ & NA \\
\hline Impedance & \multicolumn{2}{|c|}{$50 \Omega$} \\
\hline Peak power & $550 \mathrm{~kW}$ & $1 \mathrm{MW}$ \\
\hline Pulse length & $1.3 \mathrm{~ms}$ & $1.3+\mathrm{ms}$ \\
\hline Repetition Rate & $60 \mathrm{pps}$ & $60 \mathrm{pps}$ \\
\hline Average power & $48 \mathrm{~kW}$ & $60 \mathrm{~kW}$ (cooling limit) \\
\hline Bias & $\pm 2.5 \mathrm{kV}$ & $\pm 2.5 \mathrm{kV}$ \\
\hline
\end{tabular}

A simple window geometry was chosen to facilitate manufacturing and assembly. The experience of KEK in

\footnotetext{
* Supported by US DOE Contract No. DE-AC05-00OR22725 †campisi@jlab.org
}

the use of couplers of similar geometry for Tristan and KEK-B had demonstrated that high power could be reached with short conditioning times [3]. The KEK couplers had reached close to $1 \mathrm{MW}$ in $\mathrm{CW}$ during conditioning and close to $400 \mathrm{~kW}$ in operation. To adapt that design to the SNS frequency, a geometrical scaling of the coupler's main dimensions with frequency was implemented. The FPC design is shown in Figure 1.

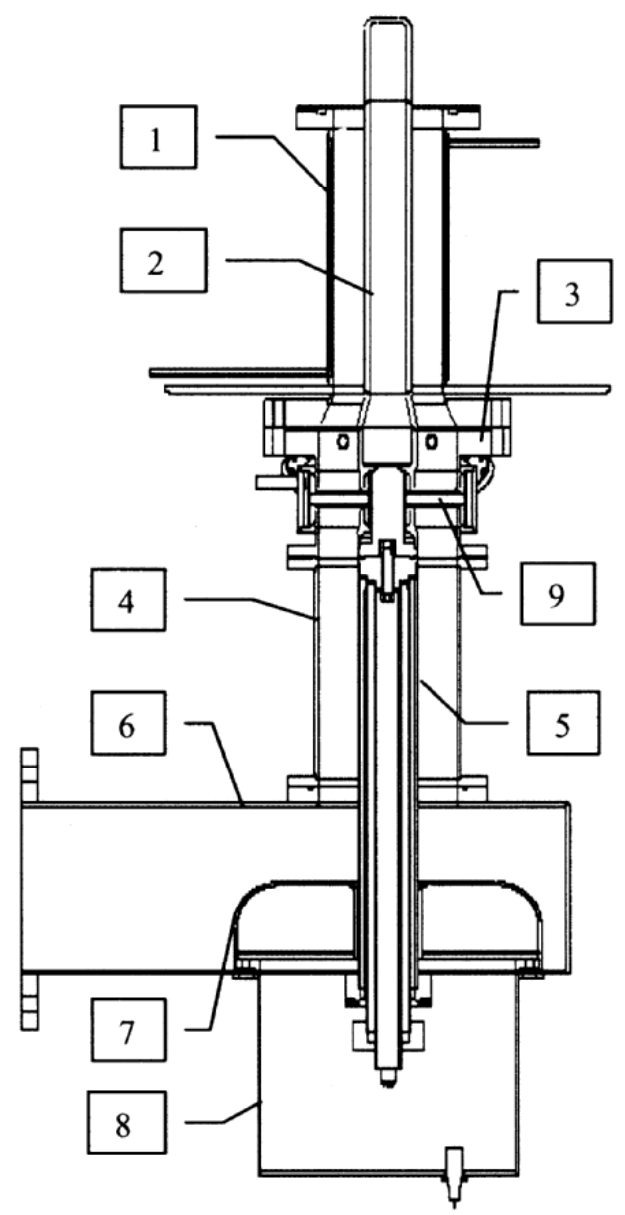

1 - Outer Conductor

3 - Window Assembly

5 - Inner Extension

7 - Doorknob

9 - Ceramic Window

Figure 1: Fundamental Power Coupler.

By this method, several properties would remain invariant, including the impedance and the multipacting
2 - Inner Conductor

4 - Outer Extension

6 - Waveguide

8 - Waveguide Cover 
levels in the coaxial part of the FPC [4]. However, some of the constraints imposed by the cryomodule geometry and assembly procedures led to a number of design modifications.

Below are discussed the main characteristics of the FPC design and some of the design calculations. The results of the preliminary tests performed on the prototype FPCs are presented elsewhere [5].

\section{ELECTRICAL AND RF DESIGN}

\subsection{Coaxial Line and Window}

The design of the SNS prototype FPCs relies on a simple coaxial line at $50 \Omega$. The planar alumina window includes impedance-matching elements $[3,6]$ as well as TiN anti-multipacting coating. This component is critical for the success of the FPC's performance, so a great deal of attention has been put into the reliability of the design and the production quality controls.

\subsection{Waveguide-Coaxial Transition}

The transition between the WR975 waveguide from the klystron and the coaxial line of the FPC is provided by a doorknob configuration [4].

\subsection{Multipacting and Voltage Bias}

Multipacting in the coaxial line and at the window can produce window limitations and failures. Extensive simulations have been performed to study the multipacting behavior of the FPC and the levels and locations have been predicted [4]. The FPC includes the possibility of biasing the inner conductor via a capacitor gap between the doorknob and the inner conductor itself at variable voltages between -2.5 and $+2.5 \mathrm{kV}$. The gap is filled with Kapton ${ }^{\circledR}$ foil, which is capable of withstanding the biasing voltage.

\subsection{Coupling}

One critical parameter which the FPC must be able to meet is the $\mathrm{Q}_{\text {ext }}$. Again, extensive simulations and measurements have been performed and reported [4].

The $\mathrm{Q}_{\text {ext }}$ can vary due to the following reasons:

- The thermal contraction of the outer conductor and the thermal expansion of the inner conductor during full power operation (as much as $48 \mathrm{~kW}$ average power). This effect is estimated to alter the $\mathrm{Q}_{\text {ext }}$ by no more than $10 \%$.

- Mechanical tolerances of the FPC's inner and outer conductors. Due to these variations, the $\mathrm{Q}_{\mathrm{ext}}$ should vary by no more that $10 \%$.

- Line mismatch in the window assembly and in the door knob transition. The total VSWR is estimated to be around 1.05, so that variation of $\mathrm{Q}_{\mathrm{ext}}$ would amount to about $5 \%$.

- Uneven field profile in the cavity. This effect is currently under study. Since few statistics are available, this effect is estimated to be around $40 \%$.

\subsection{RF Losses}

The average RF dielectric losses in the window are estimated to be around $2 \mathrm{~W}$, whereas the losses in the transmission line are $30 \mathrm{~W}$ in the inner and $3 \mathrm{~W}$ in the outer conductor, between the window and the FPC's tip. These losses can be easily handled by the cooling system described in section 4 .

\section{MECHANICAL DESIGN}

Once the FPC is fully installed, it is expected to be subjected to minimal mechanical loads other than the structural requirement that it be able to support its own weight, the thermal contractions and expansions of the outer conductor that may be induced by temperature fluctuations, and the force imposed by the pressure differential between the atmosphere and the vacuum.

The weight of the extensions, waveguide, doorknob and cover is about $9 \mathrm{~kg}$. This weight is primarily conveyed through the outer extension of the FPC to the outer conductor portion of the window and to the cryomodule. In addition, a support bracket bolted to the outside of the cryomodule will be supporting some of the weight of the FPC assembly.

The total thermal contraction of the outer conductor as it ranges from room temperature to $2 \mathrm{~K}$ is $0.4 \mathrm{~mm}$. The FPC is designed so that the compliance in the bellows between the coupler and the cryomodule will compensate for this deflection. Another load will be produced by the pressure of the atmosphere on the large flange of the outer conductor when the space inside the cryomodule is evacuated. This force, distributed about the thinnest crosssectional area of the outer conductor, would cause a negligible compressive stress of $30 \mathrm{MPa}$; the Von Mises bending stress on the beam pipe to which the FPC is attached is only $12 \mathrm{MPa}$.

During assembly, a concern is the possibility of damaging the window, which would be most likely to occur during attachment of the inner extension. However, an analysis of the assembly shows that when the inner extension is deflected, the fragile window area is not the location of the highest stresses (Figure 2); instead, noticeable yielding of the window-inner extension joint would occur before damage to the window region.

To verify that the coupler assembly is not excited by the macropulse repetition rate of the accelerator, which is 60 pps, a normal modes analysis of the assembly was performed. When the FPC is mounted in the cryomodule, the inner conductor is supported at the window and thus acts like a cantilever. The calculated fundamental frequency of the assembly is $97 \mathrm{~Hz}$, above the range of concern.

Two prototypes of the FPC have now been successfully assembled and tested under vacuum without any damage to the components. 


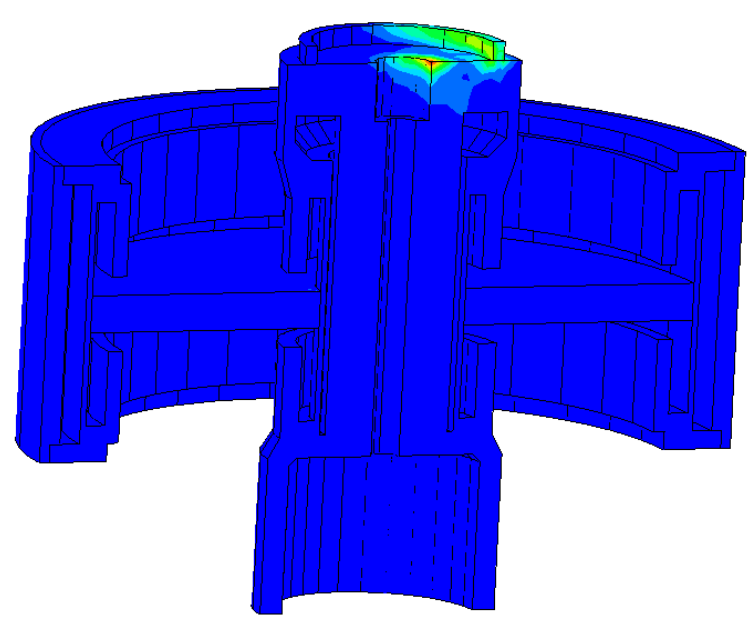

Figure 2: Stress contours on window assembly.

\section{THERMAL DESIGN}

To minimize heat transfer to the cavity beampipe, the FPC outer conductor is helium cooled. By design, the total heat load, including static and dynamic contributions, from the FPC to the $2 \mathrm{~K}$ circuit must be less than $2 \mathrm{~W}$. To handle these loads, a stream of $3 \mathrm{~atm}, 5 \mathrm{~K}$ supercritical helium with a flow rate of $0.038 \mathrm{~g} / \mathrm{s}$ per FPC removes $\sim 30 \mathrm{~W}$ of static and dynamic heating during normal operation. As shown in Figure 3, a heaterthermocouple control loop maintains the window at $300 \mathrm{~K}$ while the cold end operates at $\sim 5 \mathrm{~K}$.

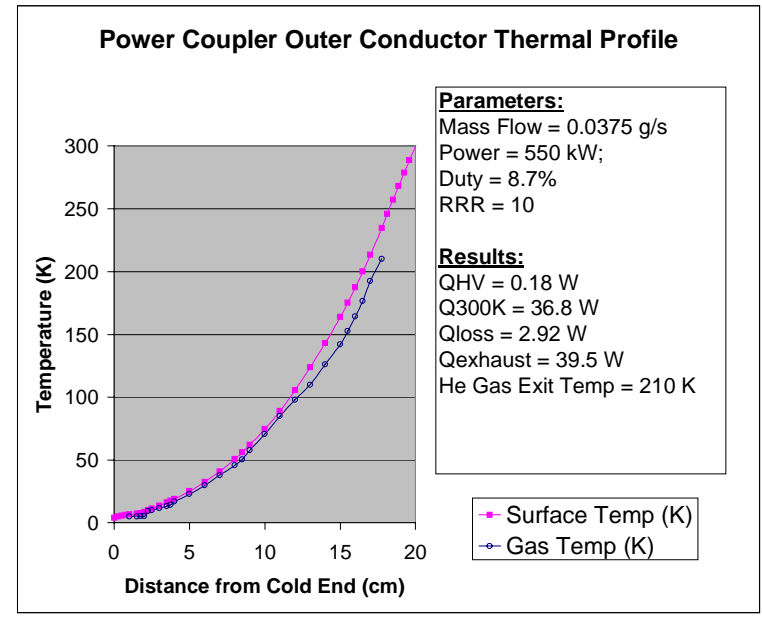

Figure 3: FPC outer conductor thermal profile.

A single helical, square-grooved flow passage is machined into a thick-walled stainless steel tube. A thinwalled stainless steel tube is then shrunk-fit over the outer diameter of the flow passages and welded leak-tight onto the ends nearest the flanges.

The internal vacuum surface is copper plated with a nominal thickness of $15 \mu \mathrm{m}(\mathrm{RRR}=10)$ to reduce resistive wall losses induced by the RF surface currents while minimally increasing the heat load to the $2 \mathrm{~K}$ circuit.

In addition, the inner extensions are water cooled to remove heat from the inner conductor and reduce radiative heating of the cavity beam pipes. A system was designed in which water enters through the outer of three concentric tubes and exits through the middle tube (the inner tube being hollow and air filled). The stream of water passes near the inner conductor-inner extension interface and removes the heat which is conducted through the inner conductor.

A thermal analysis was performed to determine the minimum acceptable water flow rate. The window was modeled axisymetrically; it was assumed that $30 \mathrm{~W}$ would be transmitted from the inner conductor to the inner extension cooling circuit, and that an additional $62 \mathrm{~W} / \mathrm{m}$ would be generated along the length of the inner extension. Initially, it was assumed that the water would be stagnant, a worst case scenario. Even with conduction the only method of heat removal, the temperature rise from the water inlet to the copper window was under $2 \mathrm{~K}$.

\section{CONCLUSIONS}

The prototype FPC for the SNS superconducting cavities is based on a design scaling from the KEK coupler. Simple changes have been implemented to adapt the FPC to the design requirements of the SNS cryomodule. Preliminary testing of the first two FPC prototypes has indicated that there are no significant design problems.

\section{ACKNOWLEDGEMENTS}

The authors wish to thank J. Henry, T. Whitlatch, B. Carpenter, K. Macha, L. King, S. Castagnola, J. Brawley, L. Turlington, R. Bundy, C. Apeldoorn, T. Elliott and W. Sommer for the essential assistance in designing and fabricating the fundamental power coupler prototypes.

\section{REFERENCES}

[1] T. E. Mason, "The Spallation Neutron Source: A Powerful Tool for Materials Research," PAC2001, Chicago, IL, June 2001.

[2] G. Ciovati et al., "Superconducting Prototype Cavities for the Spallation Neutron Source (SNS) Project," PAC2001, Chicago, IL, June 2001.

[3] S. Mitsunobu et al., "High Power Input Coupler for KEKB SC Cavity," 9th Workshop on RF Superconductivity, Santa Fe, NM, November 1999.

[4] Y. Kang et al., "Electromagnetic Simulations and Properties of the Fundamental Power Couplers for the SNS Superconducting Cavities," PAC2001, Chicago, IL, June 2001.

[5] M. Stirbet et al., "Testing Procedures and Results for the Spallation Neutron Source Fundamental Power Coupler," PAC2001, Chicago, IL, June 2001.

[6] W. R. Fowkes et al., "1.2-MW Klystron for Asymmetric Storage Ring B-Factory," PAC95, Dallas, TX, May 1995.

[7] Randall F. Barron, Cryogenic Systems. $2^{\text {nd }}$ Ed. 